\title{
Transtornos mentais comuns em profissionais de enfermagem de serviços de emergência
}

Common mental disorders in emergency services nursing professionals Trastornos mentales comunes en profesionales de enfermería de servicios de emergencia

Raysa Cristina Dias de Moura ${ }^{1}$ (1) https://orcid.org/0000-0002-5417-9179 Suzel Regina Ribeiro Chavaglia ${ }^{1}$ io https://orcid.org/0000-0001-7033-0185 Marli Aparecida Reis Coimbra ${ }^{1}$ ic https://orcid.org/0000-0001-6230-9351

Ana Paula Alves Araújo ${ }^{1}$ (D) https://orcid.org/0000-0002-5395-4559 Sabina Aparecida Scárdua ${ }^{1}$ iD https://orcid.org/0000-0002-7128-4480

Lúcia Aparecida Ferreira ${ }^{1}$ id https://orcid.org/0000-0001-6469-5444

Cíntia Machado Dutra ${ }^{1}$ id https://orcid.org/0000-0001-5120-6111 Rosali Isabel Barduchi Ohl2 id https://orcid.org/0000-0003-0369-1727

Como citar: Moura RC, Chavaglia SR, Coimbra MA, Araújo AP, Scárdua AS, Ferreira LA, et al. Transtornos mentais comuns em profissionais de enfermagem de serviços de emergência. Acta Paul Enferm. 2022;35:eAPE03032.

DOI

http://dx.doi.org/10.37689/acta-ape/2022A003032

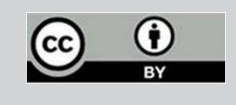

Descritores Saúde mental; Profissionais de enfermagem; Unidades hospitalares; Saúde do trabalhador; Serviços médicos

de emergência

Keywords

Mental health; Nurse practitioners; Hospital units; Occupational health; Emergency medical services

\section{Descriptores}

Salud mental; Enfermeras practicantes; Unidades hospitalarias; Salud laboral; Servicios médicos de urgencia

Submetido 14 de Outubro de 2020 Aceito 26 de Maio de 2021 Autor correspondente
Suzel Regina Ribeiro Chavaglia
E-mail: suzel.ribeiro@yahoo.com.br

Editor Associado (Avaliação pelos pares): Camila Takao Lopes (https://orcid.org/0000-0002-6243-6497) Escola Paulista de Enfermagem, Universidade Federal de São Paulo, São Paulo, SP, Brasil

\section{Resumo}

Objetivo: Analisar as variáveis sociodemográficas e de trabalho quanto ao risco de transtorno mental comum em profissionais de enfermagem que atuam em serviços de atenção às urgências e emergências.

Métodos: Estudo observacional, transversal com abordagem quantitativa, realizado no Pronto Socorro Adulto e nas Unidades de Terapia Intensiva Adulto e Coronariana de um hospital público de ensino e em duas Unidades de Pronto Atendimento públicos, do interior de Minas Gerais, Brasil. A coleta de dados ocorreu por meio de dois instrumentos: questionário sociodemográfico e profissional e o Self-Reporting Questionnaire. Para as análises foi realizada a estatística descritiva, Qui-quadrado de Pearson, exato de Fisher e regressão logística binomial.

Resultados: Participaram 302 profissionais de enfermagem. Observou-se prevalência de 20,5\% para transtornos mentais comuns. Na análise bivariada, as variáveis relacionadas aos transtornos mentais comuns foram: não ter filhos $(p=0,025)$, trabalhar nos setores da atenção terciária $(p=0,008)$, regime de contrato estatutário $(p=0,041)$. Na análise multivariada, os setores hospitalares $(p=0,001)$ e 0 cargo de enfermeiro $(p=0,017)$ indicaram risco elevado para transtornos mentais comuns.

Conclusão: As variáveis setor e cargo se comportaram como risco e apresentaram razão de chance de prevalência de 4,21 e 2,80, respectivamente, indicando que as condições de trabalho nos ambientes de urgência e emergência associadas ao cargo de enfermeiro favorecem o desenvolvimento de transtornos mentais comuns nos profissionais de enfermagem. Este estudo possibilitou conhecer a necessidade de implantação de estratégias para identificação precoce de transtornos mentais comuns e a promoção da saúde mental dos profissionais, visando a melhoria dos aspectos psicossociais nos ambientes de trabalho.

\section{Abstract}

Objective: To analyze sociodemographic and work variables regarding the risk for common mental disorders in nursing professionals working in urgent and emergency care services.

Methods: Observational, cross-sectional, quantitative study conducted in the Adult Emergency Room and Adult and Coronary Intensive Care Units of a public teaching hospital and in two public Emergency Care Units in the countryside of Minas Gerais, Brazil. Data collection was performed with use of two instruments: sociodemographic and professional questionnaire and the Self-Reporting Questionnaire. Descriptive statistics, Pearson's Chi-square, Fisher's exact and binomial logistic regression were performed in the analyzes.

Results: Participation of 302 nursing professionals. There was a prevalence of $20.5 \%$ for common mental disorders. In the bivariate analysis, the variables related to common mental disorders were: not having children $(p=0.025)$, working in tertiary care departments $(p=0.008)$, statutory contract regime $(p=0.041)$. In the multivariate analysis, the hospital departments $(p=0.001)$ and the nurse position $(p=0.017)$ indicated a high risk for common mental disorders. 
Conclusion: The variables of department and position behaved as risk and had a prevalence odds ratio of 4.21 and 2.80 , respectively, indicating that the working conditions in urgent and emergency environments associated with the nurse position favor the development of common mental disorders in nursing professionals. Through this study, it was possible to understand the need to implement strategies for the early identification of common mental disorders and the promotion of mental health among professionals, aiming at improving psychosocial aspects in work environments.

\section{Resumen}

Objetivo: Analizar las variables sociodemográficas y de trabajo respecto al riesgo de trastornos mentales comunes en profesionales de enfermería que actúan en servicios de atención de urgencias y emergencias.

Métodos: Estudio observacional, transversal, con enfoque cuantitativo, realizado en el Servicio de Urgencias Adulto y en las Unidades de Cuidados Intensivos Adulto y Coronaria de un hospital público universitario y en dos Unidades de Pronta Atención públicas del interior de Minas Gerais, Brasil. La recopilación de datos se realizó mediante dos instrumentos: cuestionario sociodemográfico y profesional y el Self-Reporting Questionnaire. Para los análisis, se utilizó la estadística descriptiva, ji cuadrado de Pearson, exacta de Fisher y regresión logística binomial.

Resultados: Participaron 302 profesionales de enfermería. Se observó prevalencia del 20,5\% de trastornos mentales comunes. En el análisis bivariado, las variables relacionadas con los trastornos mentales comunes fueron: no tener hijos $(p=0,025)$, trabajar en sectores de atención terciaria $(p=0,008)$ y el régimen de contrato estatutario $(p=0,041)$ En el análisis multivariado, los sectores hospitalarios $(p=0,001)$ y el cargo de enfermero ( $p=0,017)$ indicaron riesgo elevado de trastornos mentales comunes.

Conclusión: Las variables sector y cargo se comportan como riesgo y presentaron razón de momios de prevalencia de 4,21 y 2,80, respectivamente, lo que indica que las condiciones de trabajo en los ambientes de urgencias y emergencias asociadas al cargo de enfermero favorecen el desarrollo de trastornos mentales comunes en los profesionales de enfermería. Este estudio permitió conocer la necesidad de implementar estrategias para la identificación temprana de trastornos mentales comunes y la promoción de la salud mental de los profesionales, con el objetivo de mejorar los aspectos psicosociales en el ambiente de trabajo.

\section{Introdução}

No mundo contemporâneo, com o passar dos anos, o ambiente de trabalho se tornou mais competitivo e a assistência à saúde mais complexa, exigindo maior qualificaçáo dos trabalhadores. Vários fatores interferem no adoecimento dos profissionais da saúde, destacam-se os sociais, os individuais, assim como as condiçóes de trabalho, entretanto, as manifestações de sofrimento não se dão de maneira única para todos. As condiçóes de trabalho representam importantes fatores que determinam a saúde dos trabalhadores, podendo desencadear intenso desgaste na saúde física e mental. ${ }^{(1,2)}$

O trabalho na área da saúde representa um cenário onde diversos fatores contribuem para o adoecimento dos profissionais, seja em decorrência da complexidade da assistência, das condiçóes de trabalho, o não reconhecimento profissional, baixos salários, além das jornadas longas e exaustivas exigidas pelas instituiçốes de saúde. ${ }^{(3,4)}$

$\mathrm{O}$ atendimento em unidades de saúde que integram as redes de atençáo às urgências e emergências tem a premissa de garantir o acolhimento de casos agudos ou crônicos agudizados de caráter clínico, psiquiátrico ou traumático, bem como oferecer otimização da assistência à saúde, pois lidam com o inesperado, a instabilidade da vítima, bem como há a necessidade de execução de procedimentos imediatos, aliados ao ritmo de trabalho acelerado. ${ }^{(5,6)}$

A assistência direta ao paciente, realizada pelos trabalhadores de enfermagem que atuam em hospitais e unidades de pronto atendimento aliada às situações de tensão, pode gerar sobrecarga e estresse psíquico, provocando adoecimento do profissional com presença de sintomas físicos e psíquicos. Os estressores do ambiente de trabalho representam potenciais determinantes aos agravos de saúde do profissional de enfermagem, com o desencadeamento de transtornos mentais comuns (TMC). ${ }^{(7-10)}$

O TMC, também denominado de distúrbio psíquico menor, é uma expressão utilizada para designar sintomas não psicóticos, frequentemente, relacionados a quadros subclínicos de estresse, ansiedade e depressão que caracterizam alteração do funcionamento normal do organismo representados por insônia, irritabilidade, fadiga, dificuldade de concentração, esquecimento e queixas somáticas. ${ }^{(11,12)}$

Os sintomas entre os indivíduos variam, consideravelmente, e pode haver uma combinação entre eles. ${ }^{(13,14)}$ Além do sofrimento mental e físico são mais propensos à incapacidade para a atividade laboral, gerando redução da produtividade e desempenho, e custos adicionais às instituiçóes. ${ }^{(9,15)}$

Há evidências de que o desencadeamento deste transtorno nos profissionais de saúde tenha origem 
em diversos fatores. Dentre as causas ponta-se para o ritmo acelerado, longas e exaustivas jornadas de trabalho e intensa responsabilidade nas tarefas desempenhadas. Tudo pode interferir na qualidade da assistência, bem como no cuidado ao paciente, gerando repercussões nos indicadores organizacionais. ${ }^{(1,16)}$

O TMC associa-se ao esgotamento profissional (burnout), uma vez que prejudica os aspectos físicos e emocionais da pessoa, pelas demandas excessivas de energia. $\mathrm{O}$ ambiente de assistência ao paciente grave favorece o estresse ocupacional pela dinâmica do trabalho, pressóes das atividades e sentimentos conflituosos, como a morte. ${ }^{(9)}$

Apesar de constituírem importante problema de saúde pública mundial na atualidade, observa-se insuficiência de estudos sobre a temática na literatura científica nacional. ${ }^{(12)}$

Nesse sentido, investigar a presença destes transtornos nos profissionais de enfermagem é fundamental para subsidiar as medidas de proteção à saúde mental dos trabalhadores com enfoque no cuidado seguro e de qualidade aos pacientes e, por conseguinte, assegurar melhor funcionamento dos serviços de assistência à saúde. ${ }^{(16)}$

Considerando a relevância dessa temática para a organização e planejamento de ações de atenção à saúde mental, esta pesquisa tem como objetivo analisar as variáveis sociodemográficas e de trabalho quanto ao risco de transtorno mental comum em profissionais de enfermagem que atuam em serviços de atenção às urgências e emergências.

\section{Métodos}

Estudo observacional, transversal, com abordagem quantitativa, realizado em serviços de atenção às urgências e emergências, no interior de Minas Gerais, Brasil. As instituiçôes do estudo foram um hospital público de ensino, vinculado à Universidade Federal do Triângulo Mineiro (UFTM) e duas Unidades públicas de Pronto Atendimento (UPA). A instituição hospitalar possui 302 leitos ativos, sendo de alta complexidade e referência para 27 municípios que compóem a macrorregião de saúde Triângulo Sul, atendendo, pelo Sistema Único de Saúde - SUS, cerca de $73 \%$ de todos os casos de média e alta complexidade da macrorregiáo e $100 \%$ dos casos de alta complexidade no município, e de outros Estados do país. ${ }^{(17)}$ No hospital, a pesquisa foi realizada nos setores de Unidade de Terapia Intensiva Adulto (UTI - A), Unidade de Terapia Intensiva Coronariana (UTI - C) e Pronto Socorro Adulto (PSA).

As UPA são estabelecimentos de saúde de complexidade intermediária entre as Unidades Básicas de Saúde (UBS)/Saúde da Família e a Rede Hospitalar de atenção às urgências, de porte secundário, cada uma com, no mínimo, 11 leitos de observaçáo e capacidade de atendimento médio de 250 pacientes por dia. ${ }^{(18)}$

Incluíram-se todos os profissionais de enfermagem $(n=342)$, lotados nos serviços supracitados. Excluíram-se os trabalhadores que estavam de férias, afastados ou de licença e não retornaram durante o período de três meses da coleta de dados, ou os que coincidiram na população por trabalhar em duas das referidas instituiçóes, garantindo a participação do profissional uma única vez. Do total dos funcionários, houve perda de 11,7\% (40 sujeitos), considerando-se férias/licenças e recusas em participar, totalizando uma amostra de 302 profissionais.

Utilizaram-se dois instrumentos autoaplicáveis: Questionário Sociodemográfico e Profissional e o Self-Reporting Questionnaire (SRQ-20). As variáveis sociodemográficas do instrumento foram: sexo, idade, estado conjugal, ter filhos, cor da pele, e as variáveis profissionais investigadas foram: setor de trabalho, cargo na equipe de enfermagem, quantidade de vínculos empregatícios, regime de contrato e renda mensal.

O SRQ-20 é um instrumento criado por Harding et al, em 1980, recomendado pela Organização Mundial de Saúde (OMS) para mensuração do indicativo de TMC em estudos comunitários e atenção básica à saúde, principalmente nos países em desenvolvimento. Ele avalia queixas somáticas sem especificação, insônia, cefaleia, irritação, fadiga, lapso e dificuldade de concentração, com a validação no Brasil em 1986. ${ }^{(19)}$

O SRQ-20 é composto por 20 questóes, sendo quatro referentes a sintomas físicos e 16 sobre distúrbios psicoemocionais, para o rastreio da mor- 
bidade não psicótica, autoaplicável, com escalas dicotômicas (sim/não), de fácil compreensão e rápida aplicação. ${ }^{(20,21)}$ A pontuação deste instrumento varia de 0 a 20 , o escore total igual ou menor que 7 define caso negativo e igual ou maior que 8 considera-se positivo. ${ }^{(21)}$

O período da coleta de dados foi de outubro a dezembro de 2018 e os instrumentos de pesquisa foram aplicados face a face, pelo pesquisador e outro colaborador, devidamente, capacitado quanto aos instrumentos e abordagem dos participantes. Os trabalhadores foram abordados no local de trabalho e, após esclarecimentos da pesquisa, foi coletada a assinatura no Termo de Consentimento Livre e Esclarecido (TCLE) para em seguida responderem aos instrumentos. Os dados foram digitados em planilha eletrônica do programa Microsoft Office Excel $^{\circledR} 2013$, com dupla digitação por pessoas distintas e validação das planilhas eletrônicas.

Utilizou-se para a análise dos dados a estatística descritiva, a análise bivariada (Qui-quadrado de Pearson, exato de Fisher, razão de prevalência (RP) e razão de chance de prevalência (RCP) e análise multivariada à regressão logística binomial. Esta última foi avaliada pelo teste de Hosmer e Lemeshow $(\mathrm{p}=0,66)$, onde a não significância estatística garante consistência ao teste multivariado aplicado. Os cálculos estatísticos foram realizados por meio do software Statistic Package for Social Sciences (SPSS) versão 20.0. O nível de significância para os testes foi de $0,05 \%$.

Esta pesquisa foi aprovada pelo Comitê de Ética em Pesquisa com Seres Humanos da instituição, conforme parecer no 2.898.305/2018 e CAAE 92040318.3.0000.5154.

\section{Resultados}

Participaram da pesquisa 302 profissionais de enfermagem. A maior parte atuava nas UPA, $176(58,3 \%)$ e prevaleceu os técnicos de enfermagem com 220 $(72,8 \%)$. As variáveis sociodemográficas prevalentes foram o sexo feminino 238 (78\%), idade entre 18 e 39 anos 191 (63,2\%), cor de pele branca, 171 $(56,6 \%)$, sem companheiro $153(50,6 \%)$ e com filhos $179(59,4 \%)$ como demonstra a tabela 1 .
Tabela 1. Distribuição das variáveis sociodemográficas e profissionais dos trabalhadores de enfermagem de setores de urgência e emergência $(n=302)$

\begin{tabular}{|c|c|}
\hline Variáveis & $\mathrm{n}(\%)$ \\
\hline \multicolumn{2}{|l|}{ Sexo } \\
\hline Masculino & $64(21,2)$ \\
\hline Feminino & $238(78,8)$ \\
\hline \multicolumn{2}{|l|}{ Idade } \\
\hline 18 a 39 anos & $191(63,2)$ \\
\hline 40 a 59 anos & $106(35,1)$ \\
\hline$>60$ anos & $5(1,7)$ \\
\hline \multicolumn{2}{|l|}{ Estado conjugal } \\
\hline Com companheiro & $149(49,4)$ \\
\hline Sem companheiro & $153(50,6)$ \\
\hline \multicolumn{2}{|l|}{ Filhos* } \\
\hline Nenhum & $101(36,1)$ \\
\hline 1 ou mais & $179(63,9)$ \\
\hline \multicolumn{2}{|l|}{ Cor da pele } \\
\hline Branca & $171(56,6)$ \\
\hline Preta & $38(12,6)$ \\
\hline Amarela & $4(1,3)$ \\
\hline Parda & $89(29,5)$ \\
\hline \multicolumn{2}{|l|}{ Setores de trabalho } \\
\hline PSA e CTI & $126(41,7)$ \\
\hline UPA & $176(58,3)$ \\
\hline \multicolumn{2}{|l|}{ Cargo } \\
\hline Enfermeiro & $82(27,2)$ \\
\hline Técnico de enfermagem & $220(72,8)$ \\
\hline \multicolumn{2}{|l|}{ № vínculos empregatícios ${ }^{\star \star}$} \\
\hline 01 vínculo & $216(71,5)$ \\
\hline 02 vínculos & $84(28,5)$ \\
\hline \multicolumn{2}{|l|}{ Regime de contrato } \\
\hline Estatutário & $20(6,6)$ \\
\hline Consolidação das Leis Trabalhistas (CLT) & $282(93,4)$ \\
\hline \multicolumn{2}{|l|}{ Renda mensal| ${ }^{\star \star \star}$} \\
\hline 1 até 2 salários-mínimos & $93(30,8)$ \\
\hline 3 a 6 salários mínimos & $165(54,7)$ \\
\hline Acima de 6 salários-mínimos & $43(14,2)$ \\
\hline \multicolumn{2}{|l|}{ Risco para TMC } \\
\hline $\operatorname{Sim}$ & $62(20,5)$ \\
\hline Não & $240(79,5)$ \\
\hline
\end{tabular}

* Variável sem filhos com perda de 7,3\% ( $n=280)$; ** Variável número de vínculos com perda de $0,6 \%$ $(n=300) ;{ }^{\star \star \star}$ Variável renda com perda de $0,3 \%(n=301)$

A maioria dos profissionais, $216(71,5 \%)$, declarou um único vínculo empregatício, com regime de contrato de trabalho pela Consolidação das Leis Trabalhista (CLT), 282 (93,4\%). A renda mensal prevalente foi de seis salários-mínimos, 258 $(85,5 \%)$. Entre os profissionais da equipe de enfermagem detectou-se rastreamento positivo para TMC em 62 (20,5\%) sujeitos (Tabela 1).

A associação entre as variáveis sociodemográficas com o risco de TMC demonstrou que as variáveis sexo, faixa etária, estado conjugal e "não ter filhos" apresentaram maior relação com o desenvolvimento do TMC. Porém, apenas a variável "não ter fi- 
lhos" apresentou associação estatística significativa, $\mathrm{p}=0,025$ (Tabela 2).

Quanto às variáveis como o cargo de enfermeiro, trabalhar nos setores UTI e PS Adulto (atenção terciária) e possuir regime de contrato estatutário apresentaram maiores chances para o desenvolvimento de TMC. Houve significância estatística quanto aos setores UTI e PS Adulto, $\mathrm{p}=0,008$ e regime de contrato estatutário, $\mathrm{p}=0,041$ (Tabela 2).

Tabela 2. Associação entre variáveis sociodemográficas e profissionais e TMC entre trabalhadores de enfermagem de setores de urgência e emergência $(n=302)$

\begin{tabular}{|c|c|c|c|c|c|}
\hline \multirow[b]{2}{*}{ Variáveis } & \multicolumn{2}{|c|}{ TMC } & \multirow[b]{2}{*}{$\mathrm{RP}(\mathrm{IC})$} & \multirow[b]{2}{*}{$\mathrm{RCP}(\mathrm{IC})$} & \multirow[b]{2}{*}{$p$-value } \\
\hline & $\underset{n(\%)}{\operatorname{Sim}}$ & $\begin{array}{l}\text { Não } \\
\text { n(\%) }\end{array}$ & & & \\
\hline \multicolumn{6}{|l|}{ Sexo } \\
\hline Feminino & $54(22,7)$ & $184(77,3)$ & $\begin{array}{c}1,82(0,91- \\
3,62)\end{array}$ & $\begin{array}{c}2,05(0,92- \\
4,57)\end{array}$ & $0,073^{*}$ \\
\hline Masculino & $8(12,5)$ & $56(87,5)$ & & & \\
\hline \multicolumn{6}{|l|}{ Filhos } \\
\hline Nenhum & $33(26,8)$ & $90(73,2)$ & $\begin{array}{c}1,65(1,063 \\
-2,58)\end{array}$ & $\begin{array}{c}1,89(1,08- \\
3,33)\end{array}$ & $0,025^{\star}$ \\
\hline 1 ou mais filhos & $29(16,2)$ & $150(83,8)$ & & & \\
\hline \multicolumn{6}{|l|}{ Faixa etária } \\
\hline 49 anos ou mais & $10(24,4)$ & $31(75,6)$ & $\begin{array}{c}1,22(0,67- \\
2,21)\end{array}$ & $\begin{array}{c}1,29(0,59- \\
2,81)\end{array}$ & $0,51^{*}$ \\
\hline Até 48 anos & $52(19,9)$ & $209(80,1)$ & & & \\
\hline \multicolumn{6}{|l|}{ Estado conjugal } \\
\hline Com companheiro & $35(22,9)$ & $118(77,1)$ & $\begin{array}{c}1,26(0,80- \\
1,97)\end{array}$ & $\begin{array}{c}1,34(0,76- \\
2,35)\end{array}$ & $0,306^{*}$ \\
\hline Sem companheiro & $27(18,1)$ & $122(81,9)$ & & & \\
\hline \multicolumn{6}{|l|}{ Cargo } \\
\hline Enfermeiro & $21(25,6)$ & $61(74,4)$ & $\begin{array}{c}1,37(0,86- \\
2,17)\end{array}$ & $\begin{array}{c}1,50(0,82- \\
2,74)\end{array}$ & $0,182^{*}$ \\
\hline Téc. enfermagem & $41(18,6)$ & $179(81,4)$ & & & \\
\hline \multicolumn{6}{|l|}{ Setores } \\
\hline UTI + PSA & $35(27,8)$ & $91(72,2)$ & $\begin{array}{c}1,81(1,15- \\
2,83)\end{array}$ & $\begin{array}{c}2,12(1,20- \\
3,73)\end{array}$ & $0,008^{*}$ \\
\hline UPA & $27(15,3)$ & $149(84,7)$ & & & \\
\hline Regime de contrato & & & & & \\
\hline Estatutário & $8(40,0)$ & $12(60,0)$ & $\begin{array}{c}2,08(1,16- \\
3,76)\end{array}$ & $\begin{array}{c}2,81(1,09- \\
7,22)\end{array}$ & $0,041^{\star \star}$ \\
\hline $\begin{array}{l}\text { Consolidação das Leis } \\
\text { Trabalhistas (CLT) }\end{array}$ & $54(19,1)$ & $228(80,9)$ & & & \\
\hline
\end{tabular}

$\mathrm{Na}$ análise multivariada, ao realizar o ajuste de variáveis para a $\mathrm{RCP}(\operatorname{Exp}(\mathrm{B}))$, as variáveis setor (UTI e PSA), cargo e renda obtiveram associação estatística significante $\mathrm{p}=0,001(\operatorname{Exp}(\mathrm{B}): 4,21) ; \mathrm{p}=0,017(\operatorname{Exp}(\mathrm{B}): 2,80)$; $\mathrm{p}=0,021(\operatorname{Exp}(\mathrm{B}): 0,57)$, respectivamente. Quanto à consistência com intervalo de confiança acima de 1 , foi apresentado pelo setor (IC 95\%: 1,84-9,63) e o cargo (IC 95\%: 1,20-6,52). A renda apresentou IC 95\% variando de 0,35 a 0,92 (Tabela 3 ).
Tabela 3. Regressão logística binomial entre as variáveis sociodemográficas e profissionais e TMC entre trabalhadores de enfermagem de setores de urgência e emergência

\begin{tabular}{|c|c|c|c|c|}
\hline \multirow{2}{*}{ Variáveis } & \multirow{2}{*}{$\operatorname{Exp}(B)$} & \multicolumn{2}{|c|}{ IC $95 \% / \operatorname{Exp}(\mathrm{B})$} & \multirow{2}{*}{$p$-value } \\
\hline & & Inferior & Superior & \\
\hline Setor & 4,21 & 1,84 & 9,63 & 0,001 \\
\hline Regime de contrato & 2,38 & 0,76 & 7,44 & 0,133 \\
\hline Companhia & 1,44 & 0,79 & 2,61 & 0,227 \\
\hline Sexo & 1,93 & 0,83 & 4,46 & 0,123 \\
\hline Cargo & 2,80 & 1,20 & 6,52 & 0,017 \\
\hline Renda & 0,57 & 0,35 & 0,92 & 0,021 \\
\hline Idade & 0,99 & 0,95 & 1,02 & 0,718 \\
\hline
\end{tabular}

\section{Discussão}

Neste estudo a prevalência de TMC representou $20,5 \%$, sendo maior que um estudo de base populacional realizado com 848 mulheres por inquérito domiciliar em Campinas-SP que apresentou prevalência de $18,7 \% .^{(22)}$ Porém, outros estudos nacionais apresentaram prevalências maiores, dois deles realizados com profissionais de enfermagem em hospitais, um no hospital psiquiátrico com 25,7\% e outro com 285 trabalhadores de hospital geral do Paraná, Brasil, com 32,6\% de TMC. ${ }^{(23,24)}$

Estudo de revisão sistemática, realizado no Reino Unido, que objetivou estimar a prevalência de TMC de profissionais, evidenciou nos enfermeiros índices de TMC mais elevados em relação aos médicos, $38,2 \%$ e $31,6 \%$, respectivamente. ${ }^{(25)}$

Outra pesquisa realizada com 280 técnicos de enfermagem, de um hospital universitário no norte de Minas Gerais- Brasil, a prevalência de TMC foi de $46,9 \%$, destacando a relação entre o trabalho e a ocorrência de adoecimento por transtornos mentais nos profissionais. ${ }^{(26)}$

Pesquisa realizada com 385 profissionais de enfermagem na França reafirma essa realidade ao identificar que os enfermeiros que trabalham em Unidades de Emergência, Recuperação Pós-anestésica e UTI apresentaram alto nível de demanda e baixo nível de controle do estresse laboral, independente do gênero. Além disso, os enfermeiros com nível mais alto de qualificação sofreram maior pressão no trabalho e mais baixo nível de controle, independentemente dos ambientes de cuidados intensivos. ${ }^{(27)}$

A análise bivariada com as variáveis sociodemográficas, desta pesquisa, demonstrou que o sexo 
feminino apresentou duas vezes mais chances para TMC que o masculino embora não tenha sido significativo do ponto de vista estatístico, como encontrado noutro estudo que aplicou os testes de Qui-quadrado e Exato de Fisher. ${ }^{(28)}$ A prevalência de TMC na população feminina pode ser atribuída pela dupla ocupação de papéis diante do trabalho e os encargos da família tornando-as mais suscetíveis ao adoecimento e transtornos mentais. ${ }^{24,29)}$

A maior ocorrência de TMC entre as mulheres pode estar relacionada às responsabilidades atribuídas a elas, como o cuidado dos filhos e da família aliada às atividades profissionais com longas jornadas de trabalho e inserção em postos de trabalho mais precários. Desta forma, estão mais propensas ao estresse, ansiedade e transtornos mentais. ${ }^{(10,24,29,30)}$

Os profissionais com idade acima de 49 anos apresentaram maior risco para TMC, condizendo com pesquisa realizada na Alemanha, onde a relação entre idade e queixas psicossomáticas de trabalhadores de saúde aumentou conforme a faixa etária avançada. ${ }^{(31)}$

Quanto ao estado conjugal neste estudo, a variável ter companheiro representou maior chance de desenvolvimento de TMC, apesar de não ter havido associação estatística. Esse dado é contrário à outra pesquisa em que mulheres separadas, divorciadas ou viúvas apresentaram prevalência de transtornos mentais $67 \%$ mais elevada que mulheres casadas. ${ }^{(22)}$

Em relação à variável filhos, aqueles profissionais que não os têm apresentaram mais chance de desenvolvimento para TMC, contrário a outro estudo realizado com 3.084 trabalhadores da atenção básica e de média complexidade de cinco municípios baianos que demonstrou que os profissionais que têm filhos apresentaram maior predisposição para o TMC. ${ }^{(32)}$

Estudos reforçam essa realidade e destacam que a maior prevalência de adoecimento mental nas mulheres que possuem filhos pode ser desencadeada pela dupla jornada de trabalho, por ser atribuída à mulher a responsabilidade de cuidar dos filhos e família, além de suas atividades profissionais. ${ }^{(10,32)}$ No entanto, neste estudo, na direçáo oposta ao que aponta a literatura, a maior prevalência de TMC associou-se àqueles profissionais sem filhos.
Nas variáveis profissionais, os trabalhadores de enfermagem dos setores de UTI - A e PSA (atenção terciária) apresentaram duas vezes mais chances de indicativo para TMC que das UPA (atenção secundária), o que se justifica pela complexidade dos cuidados de enfermagem e a sobrecarga de trabalho nos hospitais. ${ }^{(12,13,27,28,33)}$

A UTI historicamente é considerada como ambiente estressante para os pacientes, familiares e profissionais de saúde. $\mathrm{O}$ estresse representado pelo setor ocorre, principalmente, por se tratar de um ambiente fechado, marcado por sentimentos de tristeza, sofrimento, imprevisibilidade, além do ritmo de trabalho acelerado, carga horária excessiva e rotinas exigentes. ${ }^{(13,33)}$

Estudo realizado em 15 hospitais poloneses com 406 enfermeiros intensivistas e 138 médicos intensivistas identificou níveis de estresse significativamente maiores nos enfermeiros, sendo que os profissionais do sexo feminino apresentaram níveis de estresse mais elevados do que o masculino. ${ }^{(33)}$

Nesta investigação, a variável regime de contrato indica que o profissional estatutário está duas vezes mais propenso ao TMC que aquele regido pela CLT. Pode-se inferir que esse profissional receba menor salário que o estatutário, uma vez que, na análise multivariada, a renda baixa se comportou como fator de proteção para TMC. Esse dado vai no sentido oposto a outro estudo que aponta que profissionais com baixa renda estão mais propensos ao desenvolvimento de TMC. ${ }^{(28)}$

Os postos de trabalho com vínculos estáveis, segundo a literatura, representam maior segurança aos profissionais, tornando-os mais protegidos em comparação com os trabalhadores temporários/não concursados. Contudo, apesar da estabilidade do vínculo, os profissionais esbarram na desvalorização profissional, baixos salários e precárias condiçóes laborais, favorecendo o adoecimento mental. ${ }^{(10,26,30)}$

A regressão logística binomial também demonstrou que os setores UTI - A e PSA e o cargo de enfermeiro são variáveis que impactaram no risco de TMC entre os profissionais de enfermagem estudados.

O ambiente laboral impacta a saúde psíquica de profissionais de enfermagem, relacionado à sobre- 
carga de trabalho, problemas com a instituição, falta de recursos materiais e mão de obra, insatisfação e prejuízo no cuidado de pacientes e também no desencadeamento de doenças como enxaqueca, estresse, hipertensão, dores osteomusculares e insônia. ${ }^{(30)}$

Estudo realizado durante a pandemia do COVID-19, em nove hospitais no Reino Unido com trabalhadores de UTI, contou com 709 participantes, sendo a maioria (344) enfermeiros e revelou, na regressão logística, que os enfermeiros eram os profissionais mais propensos a atingir os limiares de depressão (moderada e grave), provável Transtorno de Estresse Pós-Traumático e ansiedade (moderada e grave). ${ }^{(34)}$

Os setores críticos como as UTI, Centro Cirúrgico e Pronto Socorro são unidades que requerem atenção e cuidado dos trabalhadores de enfermagem, onde se ressalta a sobrecarga cotidiana de atividade laboral, prejuízos físicos e emocionais e falta de tempo para descanso. ${ }^{(35)}$

Os profissionais de enfermagem merecem atenção quanto à saúde mental, visto que são expostos a diversos tipos de estresse como decisóes rápidas e assertivas, estado de saúde crítico de pacientes por lidarem com a morte, além de questóes de relacionamentos conflituosos no trabalho, havendo necessidade de intervenção de saúde mental junto à categoria. $^{(12)}$

Os achados deste estudo ainda apontam que esses setores apresentaram 4,2 vezes mais risco de desenvolver TMC que os demais, o que corrobora com a literatura que tem apresentado os setores de cuidados intensivos e emergenciais como insalubres, de propensão ao desequilíbrio psicológico. ${ }^{(1,12,18,27,29,30,33-35)}$

Este estudo aponta como limitação dos resultados o fato de o delineamento ser transversal, não permitindo inferir causalidade, sendo os dados coletados em um único momento. Outra limitação refere-se à elegibilidade da população para a pesquisa; não foi aplicado nenhum cálculo de amostra. Também não foram verificadas nas variáveis sociodemográficas e profissionais as questôes como doenças pré-existentes, escolaridade, horário de trabalho e tempo de trabalho, não permitindo assim outras correlaçôes de variáveis.

\section{Conclusão}

A prevalência para transtornos mentais comuns foi de $20,5 \%$. Em resposta à questão de pesquisa, as variáveis setores UTI - A e PSA e o cargo de enfermeiro se comportaram como risco para indicativo de transtornos mentais comuns com razão de chance de prevalência de 4,21 e 2,80, respectivamente. As condiçôes do ambiente laboral, como a sobrecarga de trabalho, associadas às demandas psicológicas de assistência ao paciente crítico configuram o cotidiano ocupacional dos profissionais de enfermagem e podem favorecer o desenvolvimento dos transtornos mentais comuns. Este estudo alerta para atenção aos trabalhadores destas unidades, uma vez que os transtornos mentais comuns podem prejudicar o padrão de saúde mental do trabalhador e impactar, negativamente, na prática profissional, levando risco tanto a assistência ao paciente, quanto a própria saúde. Espera-se que os resultados deste estudo possam fomentar novas investigaçôes sobre as variáveis e os riscos à saúde dos trabalhadores de enfermagem que exercem suas atividades laborais nos serviços de atenção às urgências e emergências, assim como auxiliar na adoção de estratégias para a identificação precoce dos TMC e a promoção da saúde mental dos profissionais de saúde, visando o controle dos aspectos psicossociais no trabalho.

\section{Colaborações}

Moura RCD, Chavaglia SRR, Coimbra MAR, Araújo APA, Scárdua SA, Ferreira LA, Dutra CM, Ohl RIB contribuíram com a concepção e desenho do projeto, análise e interpretação dos dados; redação do artigo e revisão crítica de conteúdo intelectual importante; aprovação final da versão a ser publicada.

\section{Referências}

1. Nascimento DS, Barbosa GB, Santos CL, Martins DF, Nascimento Sobrinho CL. Prevalence of minor psychic disorders and factors associated with intensive nurses. Rev Baiana Enferm. 2019 ;33:e28091. 
2. Oliveira AM, Araújo TM. Situações de desequilíbrio entre esforçorecompensa e transtornos mentais comuns em trabalhadores da atenção básica de saúde. Trab Educ Saúde. 2018;16(1):243-62.

3. Fernandes MA, Soares LM, Silva JS. Work-related mental disorders among nursing professionals: a Brazilian integrative review. Rev Bras Med Trab. 2018;16(2):218-24. Review.

4. Silva-Costa A, Ferreira PC, Griep RH, Rotenberg L. Association between Presenteeism, Psychosocial Aspects of Work and Common Mental Disorders among Nursing Personnel. Int J Environ Res Public Health. 2020;17(18):6758.

5. Carvalho AE, Frazão IS, Silva DM, Andrade MS, Vasconcelos SC, Aquino $\mathrm{JM}$. Stress of nursing professionals working in pre-hospital care. Rev Bras Enferm. 2020;73(2):e20180660.

6. Resende MA, Silva GA, Teixeira JC. The sense of working in the urgency and emergency network: social representations of managers and health care workers. Rev Med Minas Gerais. 2018;28(Supl 5):S280503.

7. Azevedo BD, Nery AA, Cardoso JP. Occupational stress and dissatisfaction with quality of work life in nursing. Texto Contexto Enferm. 2017;26(1):e394001.

8. Zavala MO, Klijn TP, Carrillo KL. Quality of life in the workplace for nursing staff at public healthcare institutions. Rev Lat Am Enfermagem. 2016;24:e2713

9. Castro CS, Timenetsky KT, Katz M, Corrêa TD, Felício AC, Moriyama T, et al. Burnout syndrome and engagement among critical care providers: a cross-sectional study. Rev Bras Ter Intensiva. 2020;32(3):381-90.

10. Campos FM, Araújo TM, Viola DN, Oliveira PC, Sousa CC. Occupational stress and mental health in healthcare work: inequalities of gender and race. Cad Saude Colet. 2020;28(4):579-89.

11. Zenkner KV, Denardin EF, Jesus AA, Strom BR, Silva ES, Carlesso JP. Mental health of health professionals: the illness of those who are dedicated to caring for the illness of others. Res Soc Development. 2020;9(7):e916974747.

12. Nonnenmacher LL, Loiola MA, Silva F, Melo FA, Freitas RC, Almeida MS. Disorder in nursing professionals at the emergency room: systematic literature review. Rev Mult Psic. 2019;13(48 Supp 1):120-32.

13. Buselli R, Del Guerra P, Caldi F, Veltri A, Battaglia S, Baldanzi S, et al. Mental disability management within occupational health surveillance. Med Lav. 2020;111(3):232-40.

14. Oliveira AM, Araújo TM. Situations of imbalancing between stressrewards and common mental disorders in basic health care workers. Trab Educ Saúde. 2018;16(1):243-62.

15. Audi CA, Santiago SM, Andrade MG, Francisco PM. Common mental disorder among incarcerated women: a study on prevalence and associated factors. Cien Saude Colet. 2018;23(11):3587-96.

16. Gaspar FW, Zaidel CS, Dewa CS. Rates and predictors of recurrent work disability due to common mental health disorders in the United States. PLoS One. 2018;13(10):e0205170.

17. EBSERH Hospitais Universitários Federais. Sobre o HC-UFTM. Uberaba (MG): EBSERH; 2016 [citado 2020 Jan 20]. Disponível em: http://www. ebserh.gov.br/web/hc-uftm/sobre-0-hc-uftm

18. Uchimura LY, Viana AL, Silva HP, Ibañez N. Unidades de Pronto Atendimento (UPAs): características da gestão às redes de atenção no Paraná. Saúde Debate. 2015;39(107):972-83.

19. Mari JJ, Williams P. A validity study of a psychiatric screening questionnaire (SRQ-20) in primary care in the city of Sao Paulo. Br J Psychiatry. 1986;148:23-6.
20. Gonçalves DM, Stein AT, Kapczinski F. Performance of the SelfReporting Questionnaire as a psychiatric screening questionnaire: a comparativestudy with Structured Clinical Interview for DSM-IV-TR. Cad Saude Publica. 2008;24:380-90.

21. Santos KO, Araujo TM, Pinho PS, Silva AC. Avaliação de um instrumento de mensuração de morbidade psíquica: estudo de validação do self-reporting questionnaire (SRQ-20). Rev Baiana Saúde Pública. 2010;34(3):544-60.

22. Senicato C, Azevedo RC, Barros MB. Common mental disorders in adult women: identifying the most vulnerable segments. Cien Saude Colet. 2018;23(8):2543-54.

23. Sousa KH, Lopes DP, Tracera GM, Abreu AM, Portela LF, Zeitoune RC. Common mental disorders among nursing workers in a psychiatric hospital. Acta Paul Enferm. 2019;32(1):1-10.

24. Pinhatti ED, Ribeiro RP, Soares MH, Martins JT, Lacerda MR. Minor psychiatric disorders in nursing: prevalence and associated factors. Rev Bras Enferm. 2018;71(Suppl 5):2305-12.

25. Allan SM, Bealey R, Birch J, Cushing T, Parke S, Sergi G, et al. The prevalence of common and stress-related mental health disorders in healthcare workers based in pandemic-affected hospitals: a rapid systematic review and meta-analysis. Eur J Psychotraumatol. 2020;16;11(1):1810903. Review.

26. Santos FF, Brito MF, Pinho L, Cunha FO, Rodrigues-Neto JF, Fonseca $\mathrm{AD}$, et al. Common mental disorders in nursing technicians of a university hospital. Rev Bras Enferm. 2020;73(1):1-6.

27. Trousselard M, Dutheil F, Naughton G, Cosserant S, Amadon S, Dualé C, et al. Stress among nurses working in emergency, anesthesiology and intensive care units depends on qualification: a Job Demand-Control survey. Int Arch Occup Environ Health. 2016;89(2):221-9.

28. Souza LP, Barbosa BB, Silva CS, Souza AG, Ferreira TN, Siqueira LG. Prevalência de transtornos mentais comuns em adultos No contexto da atenção primária à saúde. Rev Port Enferm Saúde Mental. 2017;(18):59-66.

29. Alves AB, Arruda AJ, Silva CC, Souza MC, Fonseca LC, Santos IB, et al. Overload of work of the nursing professionals that work in the surgical center and the repercussions in the quality of life. Int Archives Med. 2017;10(30)1-9.

30. Silva AF, Robazzi ML, Dalri RC, Monteiro CA, Mendes AM. Common mental disorders among multidisciplinary team workers at a Brazilian Intensive Care Unit. Rev Iberoam Educ Investi Enferm. 2018;8(1):3646.

31. Schulz H, Zacher H, Lippke S. The Importance of Team Health Climate for Health-Related Outcomes of White-Collar Workers. Front Psychol. 2017;8:74.

32. Sousa CC, Araújo TM, Pinho OS, Freitas AM. Insatisfação com 0 trabalho em saúde: fatores associados e diferenciais de gênero. Rev Bras Saude Ocup. 2020;45:e11.

33. Kwiatosz-Muc M, Fijałkowska-Nestorowicz A, Fijałkowska M, Aftyka A, Kowalczyk M. Stress prevalence and stressors among anaesthesiology and intensive care unit workers: a multicentre survey study. Aust Crit Care. 2018;31(6):391-5.

34. Greenberg N, Weston D, Hall C, Caulfield T, Williamson V, Fong K. Mental health of staff working in intensive care during Covid-19. Occup Med (Lond). 2021;71(2):62-7.

35. Souza RF, Rosa RS, Picanço CM, Souza Junior EV, Cruz DP, Guimarães $\mathrm{FE}$, et al. Repercussões dos fatores associados à qualidade de vida em enfermeiras de unidades de terapia intensiva. Rev Salud Pública. 2018;20(4):453-9. 